\title{
En Bloc Resection as a Potential Survival Factor for Surgical Treatment of Patients with Advanced Ovarian Cancer \\ Mall Eltermaa $^{1}$, Khalid El Khalfaoui ${ }^{2}$, Adetutu Salau ${ }^{1}$, Zaher Halwani ${ }^{{ }^{*}}$ \\ ${ }^{1}$ Department of Obstetrics and Gynecology, Vivantes Humboldt Klinikum, Berlin, Germany \\ ${ }^{2}$ Department of Obstetrics and Gynecology, Evangelical Hospital Oberhausen (EKO), Oberhausen, Germany
}

\begin{abstract}
Around $60 \%$ of ovarian cancer is diagnosed in advanced stage - FIGO III or IV. Five year survival trends for women with this diagnosis have changed little, but it is stated that progression-free and overall survival is better in patients who have had a complete resection of the cancer. In order to achieve the best surgical result, one has to use "anatomical surgery": En bloc resection for advanced ovarian cancer means using optimal anatomical approach to the tumor in a situation where the entire abdomen is the surgical compartment, which is the case for advanced ovarian cancer. The rational for using en bloc resection is the reported high rate of complete debulking, which means better survival outcome. Using anatomical landmarks and systematic approach to tumor could results in smaller blood loss in a situation where the oncological disease itself can raise the risk of perioperative bleeding. Less blood loss means smaller need for blood transfusion. Perioperative blood transfusion has been shown to be a predictor of early post-operative mortality. When blood loss is smaller and the surgery is completed with optimal movements, the operating time is shorter. Operation time and the amount of blood transfusion are related to possible intestinal anastomosis problems. Shorter operation can mean the possibility to offer more radial cytoreductive surgery for elderly patients. More than half of ovarian cancer cases are diagnosed in women aged 65 and over. Unfortunately, elderly people have shown to have less chance of receiving standard therapy. En bloc resection cases in literature have shown acceptable complication rates. Reducing operation time and blood loss means in general quicker recovery, shorter hospital stay. With optimal anatomical technique being established in a center, it is possible to teach and assess the surgeons in a more optimal way. It has been shown that structured management programs affect the rate of complete debulking and overall survival.
\end{abstract}

En bloc resection could potentially be a method which allows achievement of surgical and postoperative goals in the most optimal way

\section{Introduction}

Around $60 \%$ of women are diagnosed with advanced stage ovarian cancer - FIGO stadium III or IV [1]. This means that patients presents with a tumor affecting not only small pelvic organs, but the entire abdomen, and a possibility of distant metastasis [2]. Diagnosis in advance stage also reflects in the survival numbers - according to Unites States statistics, 5-year-survival has not changed so much: in year 1975 it was $33.7 \%$ and in the year $200846.2 \%$ [3]. This article is an option statement not a systematic review looking at some aspects of en bloc resection technique as a possible potential survival factor for ovarian cancer patients.

\section{Modalities of ovarian cancer treatment}

The treatment options for ovarian malignancy are based on two strategies: surgery and chemotherapy. In 1934 Meigs suggested for the first time that primary surgical therapy is the most important part of the treatment before adding chemotherapy and radiation [4]. After that there have been trials with practice-changing results for chemotherapy and now we have entered the modern era of personalized and immunological therapy [5]. Today, the "gold standard" is primary cytoreductive surgery with adjuvant platinum-based chemotherapy [6]. The development of surgical therapy has been mostly discussion of radicality. Today the goal of the primary cytoreductive surgery with the best possible outcome and survival rates is to reach no gross residual disease [6]. The next aspect about surgical therapy for ovarian cancer has been the question of lymphadenectomy - when and how high should it be performed $[7,8]$. When it comes to specific surgical techniques, there is a gap of unanswered questions-surgical treatment is seen as a single entity, however in reality that could be very variable.

\section{Description of en bloc operative technique for ovarian cancer}

Malignant tumors have compartments where they spread, which means that they have a certain pattern of growth, e.g. which organs are infiltrated and in which order. For ovarian cancer the compartment is the entire abdominal cavity with peritoneum being the forefront border [8]. That means that the therapy of ovarian cancer should include the understanding of this characteristic and incorporation of this into the surgical thinking. En bloc resection is nothing new in oncological surgery, but this technique needs special considerations in the case of ovarian cancer. It is recommended that an en bloc resection be performed in a retrograde fashion [9]. En bloc resection could be described as follows: complete inspection of the abdominal cavity followed by development of surgical plan and resection of all visualized surgically removable tumor: en bloc retroperitoneal resection of uterus with adnexa, pelvic peritoneum and necessary

"Corresponding Author: Dr. Zaher Halwani, Department of Obstetrics and Gynecology, Vivantes Humboldt Klinikum, Berlin, Germany, Tel: (030)130121261; E-mail: zaher.halwani@vivantes.de

Citation: Eltermaa M, Khalfaoui KE, Salau A, Halwani Z (2016) En Bloc Resection as a Potential Survival Factor for Surgical Treatment of Patients with Advanced Ovarian Cancer. Int J Gynecol Clin Pract 3: 124. doi: http://dx.doi. org/10.15344/2394-4986/2016/124

Copyright: (c) 2016 Eltermaa et al. This is an open-access article distributed under the terms of the Creative Commons Attribution License, which permits unrestricted use, distribution, and reproduction in any medium, provided the original author and source are credited. 
amount of infiltrated colon, spleen, etc. If infiltrated by the tumor, the bloc could also include abdominal sidewall and diaphragmatic peritoneum. This step is followed by further resection of macroscopically diseased tissues. The opposite method would be segmental surgery, which is used with cervical cancer - a malignancy with a completely different way of spreading. Segmental surgery could be described as linear, while en bloc resection is more parallel in thinking.

\section{En bloc resection as a potential survival factor}

Our hypothesis is that en bloc resection, as a specific surgical technique/approach to tumor removal, can provide a potential survival benefit for patients with advanced stage ovarian cancer. Next section of this article provides possible reasons favoring this hypothesis. According to literature, FIGO stage of ovarian tumor is the strongest predictor of survival. [6] The next important aspect for survival benefit has been shown to be the resection level. In an analysis of 14 studies with 13949 patients the median overall survival for patients with no gross residual disease was 77.8 months compared to patients with $0.1-1 \mathrm{~cm}$ residual disease, who had median overall survival of 39.0 or 31.1 months in patients with residual tumor $>1 \mathrm{~cm}$. [6] Therefore tumor residual after debulking surgery has been proved to be the most important prognostic factor for overall survival and similarly for the progression free survival [10]. Therefore the optimal goal of the primary surgery in advanced ovarian cancer should be complete resection of the tumor [10].

One of the specific characteristic of ovarian cancer is that it is a disease of the entire peritoneal cavity, which means that spread to the pelvic structures and peritoneal surfaces of the upper abdomen is more the rule than the exception [11]. For surgical treatment this means that the removal of the tumor means usually more than just hysterectomy with bilateral salpingo-oophorectomy in order to achieve R0 resection and improve patient's outcome - the surgical activity has to move upward in the abdomen. But these radical cytoreductive procedures are, according to literature, effective for achieving complete resection and can be performed safely, while morbidity and mortality is considered acceptable [6].

In conclusion: for patients with advanced stage ovarian cancer, the survival outcome is influenced mostly by the FIGO stadium of the disease and the level of resection of the tumor.

Ovarian cancer is not the only oncological entity where en bloc resection is used. Very often this term is used with intestinal carcinomas. Colon cancer could be compared to ovarian in several ways: as a result of its local growth, it can cause adherent attachment to adjacent organs and with advanced ovarian cancer there is very often involvement to different degree of the colon. In an article published in 1991, it was concluded that colon cancer, which involves adjacent structures, should not be regarded as incurable and en bloc resection is indicated [12]. Another very interesting article about colon cancer was published already in 1987. There the authors revealed results of a retrospective study for different surgical techniques for colon cancer adherent to adjacent structures, without distant metastasis. The different treatment techniques were: standard colectomy, en bloc resection, and colectomy with separation of adherent organs. 5 year survival rates were 61 percent for en bloc resection group versus 23 percent for colectomy group. [13]
Oncological disease with some similarities to ovarian cancer, is gastric carcinoma, which historically has been usually also diagnosed with advanced stage and is known to affect the peritoneum. Diagnostically these two carcinomas, ovarian and gastric, also pose differential diagnostic difficulties, when answering which carcinoma is the primary. With gastric cancer the primary treatment has also been radical R0 resection, which gives patients best options $[14,15]$.

In a surgical oncology book [16] is given a term anatomical surgery. En bloc resection could be looked at as a perfect example of this kind of surgery. It means using anatomical compartments and spaces to move around the tumor with the most optimal way. This optimality is the key for making this technique a survival factor.

The aim of oncological surgery is to find a way around the tumor, in order not to cut into it - the surgery has to be precise in order to give the patient the best option and reduced local recurrence. [16] Surgeon has to confine the tumor to a resectable organ, organ part or en bloc with draining lymph nodes. [16] If one starts to remove organs separately, there is a very big possibility of cutting the tumor tissue, because the original borders of the organ may not be so clearly definable after of the invasion/infiltration.

Using so called anatomical surgery or in our description the use of optimal spaces/optimal moves around the tumor, can potentially mean smaller blood loss. This aspect is important with every surgical cut, but oncological disease may have biological differences which may also contribute to blood loss. Oncological patients may be at increased risk for bleeding due to chemotherapy, anticoagulant drugs, tumor-related fibrinolysis, tumor localization and vascularity, extent of disease [17]. Still, it is interesting that most bleeding associated with surgical interventions is due to poor surgical hemostasis - surgical technique [17]. Therefore analysis and improvement in surgical technique is a key-element.

Smaller blood loss means less need for blood transfusions, which means also less transfusionrelated risks. In a cohort-study about predictors of mortality within 1 year after primary surgery for ovarian cancer, advanced cancer stage had statistically significant effect for mortality within 181-360 days after surgery, but perioperative blood transfusion significantly increased mortality 0-180 days after surgery [18]. Oncological patients have been shown to be more likely transfused than non-cancer patients [17]. Another aspect about blood loss and oncological surgery is that, although cancer is usually associated with a predominant hypercoagulable state in perioperative period, the arrow can change into coagulopathy after extensive trauma, excessive volume replacement, hypothermia, hypotension and acedemia [17].

When surgeon takes optimal steps to achieve cytoreduction and the blood loss is kept as minimal as possible, the operation time could be reduced. Keeping in mind these three important aspects could give the opportunity to provide optimal surgery for older patients, who tend to have less-optimal treatment according to studies. In a population study from Denmark [19] the analysis showed that ovarian cancer was less treated in elderly - they less often had surgical treatment, especially in advanced stages. But it was shown that the survival in elderly women was improved with the use of guidelinesrecommended therapy. An interesting conclusion was that this under-treatment cannot be explained by poorer functional status, because the difference in treatment remained even after adjustment for comorbidities, so it was stated that possible factors could include patient and/or physician choices. 
Citation: Eltermaa M, Khalfaoui KE, Salau A, Halwani Z (2016) En Bloc Resection as a Potential Survival Factor for Surgical Treatment of Patients with Advanced Ovarian Cancer. Int J Gynecol Clin Pract 3: 124. doi: http://dx.doi.org/10.15344/2394-4986/2016/124

Page 3 of 4

Surgery for advanced ovarian cancer very often means intestinal surgery with anastomosis. It has been shown that long surgical time and multiple blood transfusions are also factors associated with anastomosis leak [20].

Keeping operation time, blood loss minimal, means quicker recovery and patients can be released sooner from the hospital - that means reducing the risk of nosocomial infections and also healthcare costs.

Surgical treatment of any disease means that there are three counterparts: the patients, the disease and the surgeon. Patient presents with his/her autonomy and makes the final decision about whether the operation takes place or not. Also the patient brings the age and comorbidities into the equation. The disease is a factor which we can affect very little before surgical removal - in oncology there is a question of neoadjuvant chemotherapy. But the disease influences the outcome with its biology. When it comes to surgeon, (s)he is the one whose every move, every skill should be respectful to the patient and to the disease. Systematically developed and analyzed approach to cancer could possibly allow more systematic teaching of surgeons and that could potentially lead to better overall results and perhaps, also more confidence for treating patients at either end of age spectrum. In 2011 a study was published [21]. In this article the authors stated that surgical outcome is an important prognostic factor and the only one amenable to improvement by optimization. An optimization program was introduced in 2001 and results for patients with FIGO IIb-IV from three different stages were analyzed: before the program, during and after the introduction. They showed that the rate of complete debulking rose from $33 \%$ to $62 \%$ and median overall survival rose from 26 months to 45 months. Therefore change in surgical practice mirrored in the change of survival. Another study showed the importance of surgeon's experience: patients treated by surgeons who frequently utilized radical procedures had a significantly longer median overall survival time compared to surgeons who infrequently performed radical surgery: 42 months vs 24 months [22]. A systematic review analyzed the impact of different physician and hospital characteristics on outcome in ovarian cancer patients. Discipline and sub-specialization of the primary treating physician were identified as the most important variable associated with superior outcome [23]

- $\quad 22$ patients, FIGO stage IIb-IV: median age 58.8 years. $81.8 \%$ optimal cytoreduction - of whom $59.1 \%$ complete cytoreduction. No perioperative mortality. Patients with complete cytoreduction had a better disease free survival and overall survival [24].

- 46 patients- $43.5 \%$ of primary advanced ovarian cancer patients no visible tumor at the completion of the tumor and optimal cytoreduction was achieved in $89.2 \% ; 1$ anastomosis leakage. Patients with no visible residual tumor had longer disease free survival and longer overall survival [25].

- 70 patients, median age 59 years, 74\% FIGO IIIC, 26\% FIGO IV. Median operating time $315 \mathrm{~min}$, median estimated blood loss $1200 \mathrm{~mL}, 76 \%$ of patients requiring blood transfusions [26].

- 43 patients - optimal cytoreduction was accomplished in over $70 \%$ of cases and all had complete debulking of their pelvic tumor. No postoperative leaks or fistulis. Mean postoperative hospital stay of 16 days [27].

- 65 patients - en bloc resection plus rectosigmoid resection with end-to-end anastomosis. Residual tumor nil or $<1 \mathrm{~cm}$ in 48 patients. Median postop hospital stay was 11 days. 2 patients had anastomotic leak. One surgically related mortality [28].

- pelvic retroperitoneal approach was used in 66 of 147 (45\%) consecutive patients who underwent primary surgery with intent of cytoreduction. This approach was necessary in 60 of $94(64 \%)$ patients with residual tumor less than $0.5 \mathrm{~cm}$ and contributed to achieving such a minimal residual disease in 36 of 38 (95\%) stage IIBIIIB and 58 of 109 (53\%) IIIC-IV patients. Severe morbidity, but with no long-term sequelae, occurred in six (9\%) patients. Before surgery, only ten (15\%) of these patients had a performance status grade $0-1,21$ (32\%) had grade 2 , and 35 (53\%) grade 3-4. After surgery, these figures were 52 (79\%), 14 $(21 \%)$, and 0 , respectively. The 5 -year survival rate was $37 \%$, with a median survival and follow up time of 47 months (range 4-98) and 43 months, respectively. If the proper technique is used, complete pelvic cytoreduction is always feasible and morbidity is acceptable. In our series, it was necessary to approach the pelvis retroperitoneally in $64 \%$ of optimally cytoreduced patients, which suggests that this technique has an important clinical role in the treatment of patients with advanced ovarian cancer [29].

These articles here presented used the en bloc resection, but the primary outcome for these studies was not the analysis of this technique, but mostly intestinal anastomosis outcome. Ovarian cancer treatment - radical surgical cytoreduction - is special among oncological surgery [30]. Therefore more emphasis should be placed on the technique. With chemotherapy there are regimens that have been developed, individualized changes occur. Surgical treatment on the other hand is a lot more individualized due to the three pillars: the patient's tolerance, the tumor's resectability and the surgeon. Surgical outcome is to a far extent variable and amendable for being influenced by the therapists [10].

\section{Conclusion}

Ovarian cancer is a disease of the entire abdominal cavity. Its surgical treatment needs anatomical surgery - en bloc resection can offer that approach with possible advantages for survival. In the era of conflicting results from comparison of primary cytoreductive surgery plus adjuvant chemotherapy versus neoadjuvant chemotherapy followed by surgical debulking, there is need for prospective study also comparing not just the timing of surgical treatment but the technique. The goal of every surgical incision is to provide the patient with the best possible outcome with low rate of complications systematic en bloc resection could be the key in achieving optimal cytoredution with the most optimal way for the patient, but before making any conclusions about survival we need very well-designed study of surgical technique.

\section{Competing Interests}

The author(s) declare that they have no competing interests'.

\section{References}

1. Cancer Research UK,

2. Berek JS, Crum C, Friedlander M (2015) Cancer of the ovary, fallopian tube, and peritoneum. Int J Gynaecol Obstet 131 Suppl 2: S111-122.

3. SEER Cancer Statustics Factsheets: Ovary Cancer. National Cancer Institute, Bethesda. 
Citation: Eltermaa M, Khalfaoui KE, Salau A, Halwani Z (2016) En Bloc Resection as a Potential Survival Factor for Surgical Treatment of Patients with Advanced Ovarian Cancer. Int J Gynecol Clin Pract 3: 124. doi: http://dx.doi.org/10.15344/2394-4986/2016/124

Page 4 of 4

4. Meigs JV (1934) Tumors of the female pelvic organs. New York: The Macmillan Company, USA, $533 \mathrm{p}$.

5. Kim A, Ueda Y, Naka T, Enomoto T (2012) Therapeutic strategies in epithelial ovarian cancer. J Exp Clin Cancer Res 31: 14

6. Chang SJ, Bristow RE (2012) Evolution of surgical treatment paradigms for advancedstage ovarian cancer: Redefining 'optimal' residual disease. Gynecol Oncol 125: 483-492.

7. Panici PB, Maggioni A, Hacker N, Landoni F, Ackermann S, et al. (2005) Systematic aortic and pelvic lymphadenectomy versus resection of bulky nodes only in optimally debulked advanced ovarian cancer: a randomized clinical trial. J Natl Cancer Inst 97: 560-566.

8. Sehouli J (2012) Multimodales Management des Ovarialkarzinoms. (2. Auflage), Bremen: UNI-MED, Germany, 224 p.

9. Abu-Rustum NR, Barakat RR, Levine DA (2013) Atlas of Procedures in Gynecologic Oncology. (3rd edition) Florida: CRC Press, USA, $472 \mathrm{p}$.

10. du Bois A, Reuss A, Pujade-Lauraine E, Harter P, Ray-Coquard I, et al (2009) Role of surgical outcome as prognostic factor in advanced epithelia ovarian cancer: a combined exploratory analysis of 3 prospectively randomized phase 3 multicenter trials: by the Arbeitsgemeinschaft Gynaekologische Onkologie Studiengruppe Ovarialkarzinom (AGO-OVAR) and the Groupe d'Investigateurs Nationaux Pour les Etudes des Cancers de I'Ovaire (GINECO). Cancer 115: 1234-1244.

11. Wharton JT, Edwards CL (1983) The role of surgery in the management of gynecologic malignancies. Cancer 51: 2480-2484.

12. Kroneman $\mathrm{H}$, Castelein A, Jeekel J (1991) En bloc resection of colon carcinoma adherent to other organs: an efficacious treatment? Dis Colon Rectum 34: 780-783

13. Hunter JA, Ryan JA Jr, Schultz P (1987) En bloc resection of colon cancer adherent to other organs. Am J Surg 154: 67-71.

14. Martin RC, Jaques DP, Brennan MF, Karpeh M (2002) Achieving RO resection for locally advanced gastric cancer: is it worth the risk of multiorgan resection? J Am Coll Surg 194: 568-577.

15. Choi YY, Noh SH1,2, Cheong JH2,3, et al. (2015) Evolution of Gastric Cancer Treatment: From the Golden Age of Surgery to an Era of Precision Medicine. Yonsei Med J 56: 1177-1185.

16. Baumann M (ed) (2016) Oxford Textbook of Oncology. (3rd edition), Oxford: Oxford University Press, UK, 832 p.

17. Cata JP, GottumukkalaV (2012) Blood Loss and Massive Transfusion in Patients Undergoing Major Oncological Surgery: What Do We Know? ISRN Anesthesiology 2012

18. Ørskov M, lachina M, Guldberg R, Mogensen O, Mertz Nørgård B (2016) Predictors of mortality within 1 year after primary ovarian cancer surgery: a nationwide cohort study. BMJ Open 6: e010123.

19. Fourcadier E, Trétarre B, Gras-Aygon C, Ecarnot F, Daurès JP, et al. (2015) Under-treatment of elderly patients with ovarian cancer: a population based study. BMC Cancer 15: 937.

20. Mourton SM, Temple LK, Abu-Rustum NR, Gemignani ML, Sonoda Y, et al. (2005) Morbidity of rectosigmoid resection and primary anastomosis in patients undergoing primary cytoreductive surgery for advanced epithelial ovarian cancer. Gynecol Oncol 99: 608-614.

21. Harter P, Muallem ZM, Buhrmann C, Lorenz D, Kaub C, et al. (2011) Impact of a structured quality management program on surgical outcome in primary advanced ovarian cancer. Gynecol Oncol 121: 615-619.

22. Aletti GD, Dowdy SC, Gostout BS, Jones MB, Stanhope CR, et al. (2006) Aggressive surgical effort and improved survival in advanced-stage ovarian cancer. Obstet Gynecol 107: 77-85.

23. du Bois A, Rochon J, Pfisterer J, Hoskins WJ (2009) Variations in institutional infrastructure, physician specialization and experience, and outcome in ovarian cancer: a systematic review. Gynecol Oncol 112: 42236.

24. Yildirim Y, Ertas IE, Nayki U, Ulug P, Nayki C, et al. (2014) En-bloc pelvic resection with concomitant rectosigmoid colectomy and immediate anastomosis as part of primary cytoreductive surgery for patients with advanced ovarian cancer. Eur J Gynaecol Oncol 35: 400-407.
25. Park JY, Seo SS, Kang S, Lee KB, Lim SY, et al. (2006) The benefits of low anterior en bloc resection as part of cytoreductive surgery for advanced primary and recurrent epithelial ovarian cancer patients outweigh morbidity concerns. Gynecol Oncol 103: 977-984.

26. Mourton SM, Temple LK, Abu-Rustum NR, Gemignani ML, Sonoda Y, et al. (2005) Morbidity of rectosigmoid resection and primary anastomosis in patients undergoing primary cytoreductive surgery for advanced epithelial ovarian cancer. Gynecol Oncol 99: 608-614.

27. Bridges JE, Leung Y, Hammond IG, McCartney AJ (1993) En bloc resection of epithelial ovarian tumors with concomitant rectosigmoid colectomy: the KEMH experience. Int J Gynecol Cancer 3: 199-202.

28. Obermair A, Hagenauer S, TamandI D, Clayton RD, Nicklin JL, et al. (2001) Safety and efficacy of low anterior en bloc resection as part of cytoreductive surgery for patients with ovarian cancer. Gynecol Oncol 83: 115-120.

29. Benedetti-Panici P, Maneschi F, Scambia G, Cutillo G, Greggi S, et al (1996) The pelvic retroperitoneal approach in the treatment of advanced ovarian carcinoma. Obstet Gynecol 87: 532-538.

30. Markman M (2007) Concept of optimal surgical cytoreduction in advanced ovarian cancer: a brif critique and a call for action. J Clin Oncol 25: 41684170. 